\title{
TD-DFT and Experimental Methods for Unraveling the Energy Distribution of Charge-Transfer Triplet/Singlet States of a TADF Molecule in a Frozen Matrix
}

\author{
Seung-Je Woo and Jang-Joo Kim* \\ Department of Materials Science and Engineering, Seoul National University, Seoul 08826, Republic of Korea \\ KEYWORDS: TADF, TD-DFT, charge-transfer, triplet, energy distribution, conformational distribution.
}

\begin{abstract}
Reverse intersystem crossing (RISC) rate of a thermally activated delayed fluorescence (TADF) molecule is sensitive to the energy alignment of singlet charge-transfer state $\left({ }^{1} \mathrm{CT}\right)$, triplet charge-transfer state $\left({ }^{3} \mathrm{CT}\right)$, and locally excited triplet state $\left({ }^{3} \mathrm{LE}\right)$. However, the energy distribution of the charge-transfer states originating from the conformational distribution of TADF molecules in a solid matrix inevitably generated during the preparation of a solid sample due to the rotatable donor-acceptor linkage is rarely considered. Moreover, the investigation of the energy distribution of the ${ }^{3} \mathrm{CT}$ state is both theoretically and experimentally difficult due to the triplet instabilities of time-dependent density functional (TD-DFT) calculations and difficulties in phosphorescence measurements, respectively. As a result, the relation between conformational distribution, configurations of excited state transition orbitals, and excited state energies/dynamics have not been clearly explained. In this work, we determined the energy distribution of CT states of the TADF emitter TPSA in frozen toluene at 77 $\mathrm{K}$ by the measurement of time-resolved spectra in the full time range ( $1 \mathrm{~ns} \sim 30 \mathrm{~s}$ ) of emission including prompt fluorescence, TADF, ${ }^{3} \mathrm{CT}$ phosphorescence, and ${ }^{3} \mathrm{LE}$ phosphorescence. We obtained the energy band of CT states where ${ }^{1} \mathrm{CT}$ and ${ }^{3} \mathrm{CT}$ states are distributed in the range of 2.85-3.00 eV and 2.64-2.96 eV, respectively. We tested various global hybrid and long-range corrected functionals for the TD-DFT calculation of ${ }^{3} \mathrm{CT}$ energy of TPSA and found that only the M11 functional shows consistent results without triplet instability. We performed TD-DFT with the M11* functional optimized for robust dihedral angle scan of ${ }^{3} \mathrm{CT}$ states without triplet instability and reproduced the energy band structure obtained from the experiment. Through TD-DFT and experimental investigations, it is estimated that the dihedral angle of donor-acceptor $\left(\theta_{\mathrm{D}-\mathrm{A}}\right)$ and acceptor-linker $\left(\theta_{\mathrm{A}}\right)$ of TPSA in frozen toluene lie within the range of $70^{\circ} \leq \theta_{\mathrm{D}-\mathrm{A}} \leq 90^{\circ}$ and $0^{\circ} \leq \theta_{\mathrm{A}} \leq 30^{\circ}$ respectively. Our results show that the dihedral angle distribution must be considered for further investigation of the photophysics of TADF molecules and the development of stable and efficient TADF emitters.
\end{abstract}

\section{INTRODUCTION}

Utilizing electrically generated triplet excitons is critical to the efficiency of organic light-emitting diodes (OLEDs). ${ }^{1}$ Thermally activated delayed fluorescence (TADF) emitters have attracted great attention in the field of OLEDs due to their ability to convert nonradiative triplets into radiative singlet excitons which makes it possible to achieve internal quantum efficiency (IQE) of 100\%. Many TADF emitters have been developed since the first invention ${ }^{2}$ of organic TADF OLEDs in 2011 to replace pricy phosphorescence emitters and inefficient fluorescence emitters. ${ }^{3-10}$ However, no commercially available TADF emitters have been developed due to their poor stability ${ }^{11-15}$ and large efficiency roll-off ${ }^{16-18}$ resulting from the long lifetime of reactive triplet excited states of TADF molecules. ${ }^{19}$

Fast reverse intersystem crossing (RISC) rate of TADF emitters are required to reduce triplet lifetime. ${ }^{12-14}$ Previously, researches focused on designing TADF molecules with highly tilted donor-acceptor (D-A) structure to minimize energy gap $\left(\Delta \mathrm{E}_{\mathrm{ST}}\right)$ of $\mathrm{S}_{1}$ charge transfer $\left({ }^{1} \mathrm{CT}\right)$ state and $\mathrm{T}_{1}$ charge transfer $\left({ }^{3} \mathrm{CT}\right)$ state for faster RISC rate. ${ }^{20-24}$ However, increasing $\mathrm{D}-\mathrm{A}$ dihedral angle towards $90^{\circ}$ not only reduces the energy barrier for the RISC process but also reduces the spin-orbit coupling (SOC) that induces spin flip. Especially, the SOC of ${ }^{1} \mathrm{CT}$ and ${ }^{3} \mathrm{CT}$ states of TADF molecules with orthogonal D-A structure such as acridine donor-based ones are too weak for typical RISC rate $\left(10^{4}\right.$ $\sim 10^{6} \mathrm{~s}^{-1}$ ) indicating the presence of another RISC pathway. ${ }^{12,25-32}$ Recent researches emphasize that locally excited triplet ( $\left.{ }^{3} \mathrm{LE}\right)$ or hybrid local and charge-transfer state $\left({ }^{3} \mathrm{HLCT}\right)$ that has sufficient SOC with the ${ }^{1} \mathrm{CT}$ state must exist for efficient RISC process. ${ }^{12,30-34}$ In addition, the energy difference between ${ }^{1} \mathrm{CT},{ }^{3} \mathrm{CT},{ }^{3} \mathrm{HLCT}$, and ${ }^{3} \mathrm{LE}$ should also be minimized for a faster RISC rate. For example, Adachi et al. enhanced RISC rate by reducing ${ }^{3} \mathrm{HLCT}-{ }^{3} \mathrm{LE}$ gap and Monkman et al. enhanced the RISC rate by reducing ${ }^{3} \mathrm{CT}-{ }^{3} \mathrm{LE}$ gap. ${ }^{12,34}$ These reports were successful in enhancing RISC, but the excited states involved in the investigation were limited to discrete states at relaxed geometries, ${ }^{12,30-34}$ not considering the conformational distribution of TADF molecules in solid organic films inevitably generated $18,28,35,36$ during thermal deposition due to the rotatable D-A linkage. 

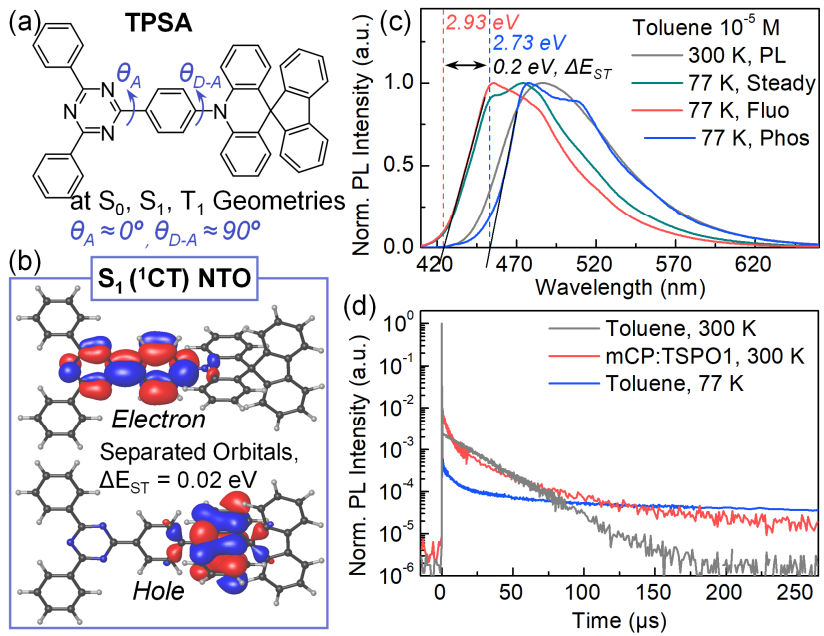

Figure 2. (a) Molecular structure of TPSA. (b) NTO of $\mathrm{S}_{1}$ state of TPSA. (c) Emission spectra of TPSA in toluene. $300 \mathrm{~K}$ : PL. $77 \mathrm{~K}$ : PL (steady state), fluorescence ( $<10 \mathrm{~ms}$ ), and phosphorescence (100 ms). (d) Transient PL decay of TPSA in toluene $\left(10^{-5} \mathrm{M}\right)$ and in mCP:TSPO1 film (10 wt $\%$ ).

Recently, we found that conformational distribution and restricted geometry relaxation of TADF molecules in solids induce different pathways for the RISC process in each conformer. ${ }^{36}$ In addition, it has been pointed out by several reports that multiexponential decay and spectral shift over time are the evidence of the conformational distribution of TADF molecules in solids. ${ }^{28,37-43}$ However, the relation of conformational distribution, configurations of excited state transition orbitals, and excited state energies have not been clearly explained. Moreover, while ${ }^{1} \mathrm{CT}$ states are widely investigated through time-resolved spectroscopy of photoluminescence (PL) in the full time range of fluorescence, triplet states are mostly investigated by PL measurement at a fixed delay time due to difficulties in phosphorescence measurement at room temperature and long-lasting phosphorescence emission up to several tens of seconds. Furthermore, some reports consider ${ }^{1} \mathrm{CT}$ and ${ }^{3} \mathrm{CT}$ as degenerated states, not considering the conformational distribution of ${ }^{3} \mathrm{CT}$ states. ${ }^{33,34}$

Time-dependent density functional theory (TD-DFT) calculation is a primary tool in predicting and investigating excited state configuration, excited state energy, and molecular geometry of TADF molecules. ${ }^{30,36,44-49}$ As with the experimental investigation of TADF molecules, most of the research focuses on excited state configurations and energies of relaxed geometry of excited states and explain experimental phenomena with discrete geometries or excited states such as ${ }^{1} \mathrm{CT},{ }^{3} \mathrm{CT}$, and ${ }^{3} \mathrm{LE}$ states. Some researches attempted to relate $D$-A dihedral angle with $S_{1}$ and $\mathrm{T}_{1}$ energies, but they have several limitations; (1) a large discrepancy with experimental energies due to inappropriate selection of functional, (2) lack of explanation to the effect of dihedral angle to excited state configurations or energies of $S_{1} / T_{1}$ states, or (3) mixed level of theory for $\mathrm{S}_{1}$ and $\mathrm{T}_{1}$ leading to inverted $\mathrm{S}_{1} / \mathrm{T}_{1}$ energies. ${ }^{33,50-52}$ These problems arise from difficulties in handling triplet states with TD-DFT due to the so-called triplet instability stemming from the exchange-interaction-sensitive nature of triplet states. ${ }^{53-56}$
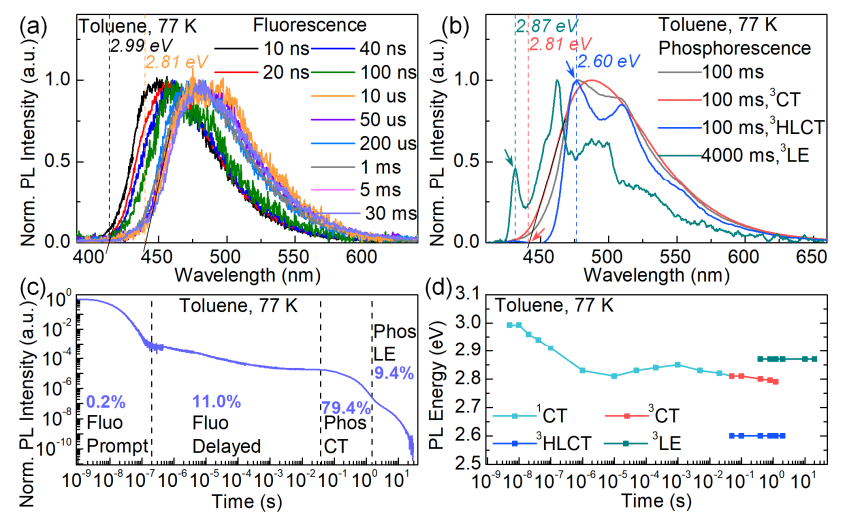

Figure 1. (a) Time-resolved PL spectra of TPSA in frozen toluene at $77 \mathrm{~K}, 337 \mathrm{~nm}$ excitation. (b) Phosphorescence of TPSA at $100 \mathrm{~ms}$ and $4000 \mathrm{~ms}$ obtained with $325 \mathrm{~nm}$ excitation. ${ }^{3} \mathrm{CT}$ and ${ }^{3} \mathrm{HLCT}$ spectra are deconvoluted from the phosphorescence spectrum at $100 \mathrm{~ms}$ (Fig. S6). (c) Decay curve of total PL emission of TPSA. (d) Changes of emission energy and emission type of PL over time. Emission energy of ${ }^{1} \mathrm{CT} /{ }^{3} \mathrm{CT}$ states and ${ }^{3} \mathrm{HLCT} /{ }^{3} \mathrm{LE}$ states are determined from the spectra onset and $1^{\text {st }}$ vibronic peak respectively.

Herein, we report experimental and theoretical investigation of the energy distribution of triplet and singlet charge-transfer states of TPSA, a TADF molecule comprised of triphenyltriazine acceptor and spiroacridinefluorene donor (Figure 1). ${ }^{22}$ To determine both the ${ }^{3} \mathrm{CT}$ and ${ }^{1} \mathrm{CT}$ energy distributions, we measured the time-resolved PL spectra of a frozen toluene solution of TPSA at $77 \mathrm{~K}$. It turns out that excited state dynamics is much more complex than a simple explanation based on three levels of ${ }^{1} \mathrm{CT},{ }^{3} \mathrm{CT}$, and ${ }^{3} \mathrm{LE}$. In the fluorescence and TADF region (1 ns $30 \mathrm{~ms}$ ), TPSA exhibits spectral shift. In the phosphorescence region (10 ms 30 s), TPSA shows a change in emission type from ${ }^{3} \mathrm{CT}$ to ${ }^{3} \mathrm{HLCT}{ }^{30,36}$ and ${ }^{3} \mathrm{LE}$. A unique electronic band structure of ${ }^{1} \mathrm{CT}$ and ${ }^{3} \mathrm{CT}$ overlapping each other were obtained from the experiments. To explain the origin of the electronic band structure of CT states, various functionals with various amounts of exact exchange were tested to find optimal functional that performs robust TD-DFT calculation of ${ }^{3} \mathrm{CT}$ s without triplet instability for the dihedral angle scan of CT state energies and NTOs of TPSA. We found that the distribution of the dihedral angle between donor-acceptor $\left(\theta_{D-A}\right)$ and dihedral angle within the acceptor $\left(\theta_{A}\right)$ was the origin of the energy band of CT states of TPSA. Furthermore, the energy distribution of ${ }^{1} \mathrm{CT}$ and ${ }^{3} \mathrm{CT}$ states calculated from TD-DFT using optimized M11* functional matched well with the experimentally determined energy distribution of ${ }^{1} \mathrm{CT} /{ }^{3} \mathrm{CT}$ states.

\section{RESULTS AND DISCUSSION}

Figure 1a shows the optimized geometries of TPSA. For all states including $\mathrm{S}_{0}, \mathrm{~S}_{1}$, and $\mathrm{T}_{1}$, the dihedral angle between the donor and acceptor is $90^{\circ}$. Hence, the electron and hole orbitals of the CT state are separated leading to almost degenerate ${ }^{1} \mathrm{CT} /{ }^{3} \mathrm{CT}$ states and a small $\Delta \mathrm{E}_{\text {ST }}$ of $0.02 \mathrm{eV}$. The $\Delta \mathrm{E}$ st obtained from the TD-DFT calculation seems to be reasonable when compared with the experimental $\Delta \mathrm{E}_{\text {ST }}$ determined from fluorescence at $300 \mathrm{~K}$ and phosphorescence at $77 \mathrm{~K}$ (Figure 1c), which is done by most of the TADF research to determine $\Delta \mathrm{E}_{\text {sт. }}$. However, determining $\Delta \mathrm{E}_{\text {ST }}$ from fluorescence and phosphorescence 
at different temperature does not involve the polarity change of solvents, leading to an underestimated $\Delta \mathrm{E}_{\text {ST }}$. Hence, $\Delta \mathrm{E}_{\text {sт }}$ should be determined from fluorescence and phosphorescence at the same temperature, which is $0.2 \mathrm{eV}$ for TPSA (Figure 1c). Experimentally determined $\Delta \mathrm{E}_{S \mathrm{~T}}$ is much larger than the $\Delta \mathrm{EST}_{\mathrm{ST}}$ calculated from TD-DFT calculation, indicating that the real TPSA molecules in frozen toluene are not in an orthogonal D-A geometry as predicted from the TD-DFT geometry optimization. The PL decay of TPSA in toluene at $300 \mathrm{~K}$ can be perfectly fitted with a biexponential decay function and shows no spectral shift because the TADF molecules can relax completely before emission or intersystem crossing (ISC) occurs at any excited states due to freely moving solvent molecules (Figure 1d, S1). ${ }^{36}$ However, PL decay of TPSA in frozen toluene or doped in solid mCP:TSP01 film shows multiexponential decay and spectral shift over time because geometry relaxation of TPSA is restricted in solid matrix. ${ }^{18,36}$ Hence, the conformational distribution of TADF molecules must be considered to investigate RISC mechanism of TADF molecules in solids.

Energy distribution of ${ }^{1} \mathrm{CT}$ state of TPSA can be determined from the time-resolved fluorescence spectra (Figure 2a). From $10 \mathrm{~ns}$ to $30 \mathrm{~ms}$, the emission changes from prompt fluorescence to delayed fluorescence and exhibits spectral redshift followed by blueshift (Figure 2a, d). The spectral shift over time stems from the difference of radiative decay rate and RISC rate caused by the different conformations (Figure S2). ${ }^{36,40,41}$ The emission energy of ${ }^{1} \mathrm{CT}$ is the highest at $10 \mathrm{~ns}$ for $2.99 \mathrm{eV}$ and lowest at $10 \mathrm{us}$ for $2.81 \mathrm{eV}$. Energy distribution of ${ }^{3} \mathrm{CT}$ state of TPSA can also be determined from the time-resolved phosphorescence spectra (Figure 2b,d, and S3-S6). Phosphorescence of TPSA changes from broad ${ }^{3} \mathrm{CT}$ type emission (prompt phosphorescence) to featured ${ }^{3} \mathrm{LE}$ emission (delayed phosphorescence), exhibiting dual phosphorescence (Figure S4). The ${ }^{3} \mathrm{LE}$ type emission is from the donor (spiroacridinefluorene, SAF), and its energy determined from the $1^{\text {st }}$ vibronic peak is $2.87 \mathrm{eV}$ (Figure $2 \mathrm{~b}, \mathrm{~S} 3$ ). The ${ }^{3} \mathrm{LE}$ emission is also observed from phosphorescence at $405 \mathrm{~nm}$ excitation which is only absorbed by the CT state of TPSA (Figure S3). Hence, the ${ }^{3}$ LE state observed from the delayed phosphorescence is generated by intersystem crossing from ${ }^{1} \mathrm{CT}$ to ${ }^{3} \mathrm{CT}$ followed by intramolecular triplet energy transfer (ITET) from ${ }^{3} \mathrm{CT}$ to ${ }^{3} \mathrm{LE}\left({ }^{1} \mathrm{CT} \rightarrow{ }^{3} \mathrm{CT} \rightarrow{ }^{3} \mathrm{LE}\right) .{ }^{57}$ Therefore, the energy level of the ${ }^{3} \mathrm{CT}$ state of some conformers of TPSA is higher than that of the ${ }^{3} \mathrm{LE}$ SAF state. On the other hand, prompt phosphorescence is comprised of ${ }^{3} \mathrm{CT}$ states of different conformers instead of a ${ }^{3} \mathrm{CT}$ state of a single geometry (Figure S5). The prompt phosphorescence at $100 \mathrm{~ms}$ can be deconvoluted with featureless ${ }^{3} \mathrm{CT}$ emission and hybrid local and chargetransfer ( $\left.{ }^{3} \mathrm{HLCT}\right)$ emission with three major peaks $(476 / 510 / 550 \mathrm{~nm})$. The energy of ${ }^{3} \mathrm{CT}$ and ${ }^{3} \mathrm{HLCT}$ determined from the deconvoluted phosphorescence spectra at $100 \mathrm{~ms}$ is $2.81 \mathrm{eV}$ and $2.60 \mathrm{eV}$ respectively. Hence, the minimum energy of ${ }^{3} \mathrm{CT}$ is $2.60 \mathrm{eV}\left({ }^{3} \mathrm{HLCT}\right)$ and the maximum energy of ${ }^{3} \mathrm{CT}$ is higher than $2.87 \mathrm{eV}\left({ }^{3} \mathrm{LE}\right)$. As shown in Figure 2d, some triplet states of TPSA have higher energy than some singlet states. According to Hund's multiplicity rule, the triplet energy is always lower than the singlet state of a single molecule. Therefore, it is clear that

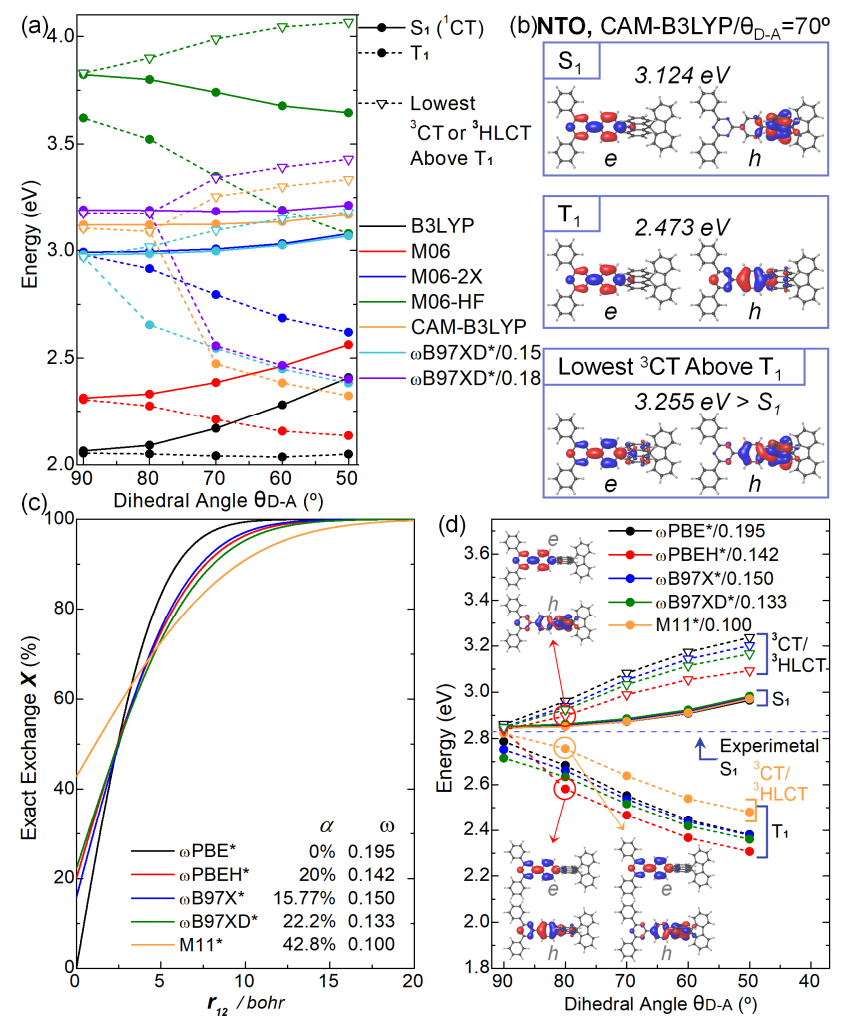

Figure 3 TD-DFT calculation results of TPSA. (a) Energies of $\mathrm{S}_{1}$ $\left({ }^{1} \mathrm{CT}\right), \mathrm{T}_{1}$, and lowest ${ }^{3} \mathrm{CT}\left({ }^{3} \mathrm{HLCT}\right)$ state above $\mathrm{T}_{1}$ as a function of the dihedral angle between the donor and linker $\left(\theta_{D-A}\right)$. (b) NTOs of $\mathrm{S}_{1}\left({ }^{1} \mathrm{CT}\right), \mathrm{T}_{1}$, and ${ }^{3} \mathrm{CT}$ states at $\theta_{\mathrm{D}-\mathrm{A}}=70^{\circ}$, calculated with CAM-B3LYP functional.(c) Amounts of exact exchange $(\boldsymbol{X})$ included in the modified long-range corrected functionals as a function of interelectronic distance $\left(r_{12}\right) . \quad \boldsymbol{\alpha}\left(\boldsymbol{X}\right.$ at $\left.r_{12}=0\right)$ and modified range separation parameters $(\omega)$ for the functionals are shown in the figure. (d) Energies of $\mathrm{S}_{1}, \mathrm{~T}_{1}$, and lowest ${ }^{3} \mathrm{CT}$ or ${ }^{3}$ HLCT state above $T_{1}$ as a function of $\theta_{D-A}$ using various functionals with optimized range separation parameters $(\omega)$. The $\omega$ parameters were optimized to match the experimental $\mathrm{S}_{1}$ energy of TPSA at $\theta_{\mathrm{D}-\mathrm{A}}=90^{\circ}$. Lowest ${ }^{3} \mathrm{CT}$ or ${ }^{3}$ HLCT states above $\mathrm{T}_{1}$ states are indicated as ${ }^{3} \mathrm{CT} /{ }^{3} \mathrm{HLCT}$. NTOs for triplet states calculated with $\omega \mathrm{PBEH}^{*}$ and $\mathrm{M} 11^{*}$ at $\theta \mathrm{D}-\mathrm{A}=80^{\circ}$ are shown.

the conformational distribution of TPSA is present in frozen toluene. Since the spiro-linked fluorene works as a rigid scaffold that prevents the crooking of the acridine moiety, ${ }^{22,58}$ the rotation of bonds within the acceptor or the dihedral angle between the donor and acceptor should be the origin of the conformational distribution TPSA.

To further investigate the energy distribution of the TADF molecules, time-dependent density functional theory (TDDFT) calculations for energies of ${ }^{1} \mathrm{CT}$ and ${ }^{3} \mathrm{CT}$ as a function of the dihedral angle between the donor and acceptor $\left(\theta_{D-A}\right)$ was considered first. As the CT energies and triplet states are sensitive to the amount of exact exchange $(\boldsymbol{X})$, the functional should be optimized for the TD-DFT calculation of TADF molecules. ${ }^{48,53-56,59,60}$ There are several reports of theoretical calculation of $S_{1}$ and $T_{1}$ states of TADF molecules as a function of the $\mathrm{D}-\mathrm{A}$ dihedral angle. A calculation result ${ }^{33}$ performed with the B3LYP functional shows the increase of both the $\mathrm{S}_{1}$ and $\mathrm{T}_{1}$ state by the decrease of the dihedral angle due to the small amount of global exact exchange. In 

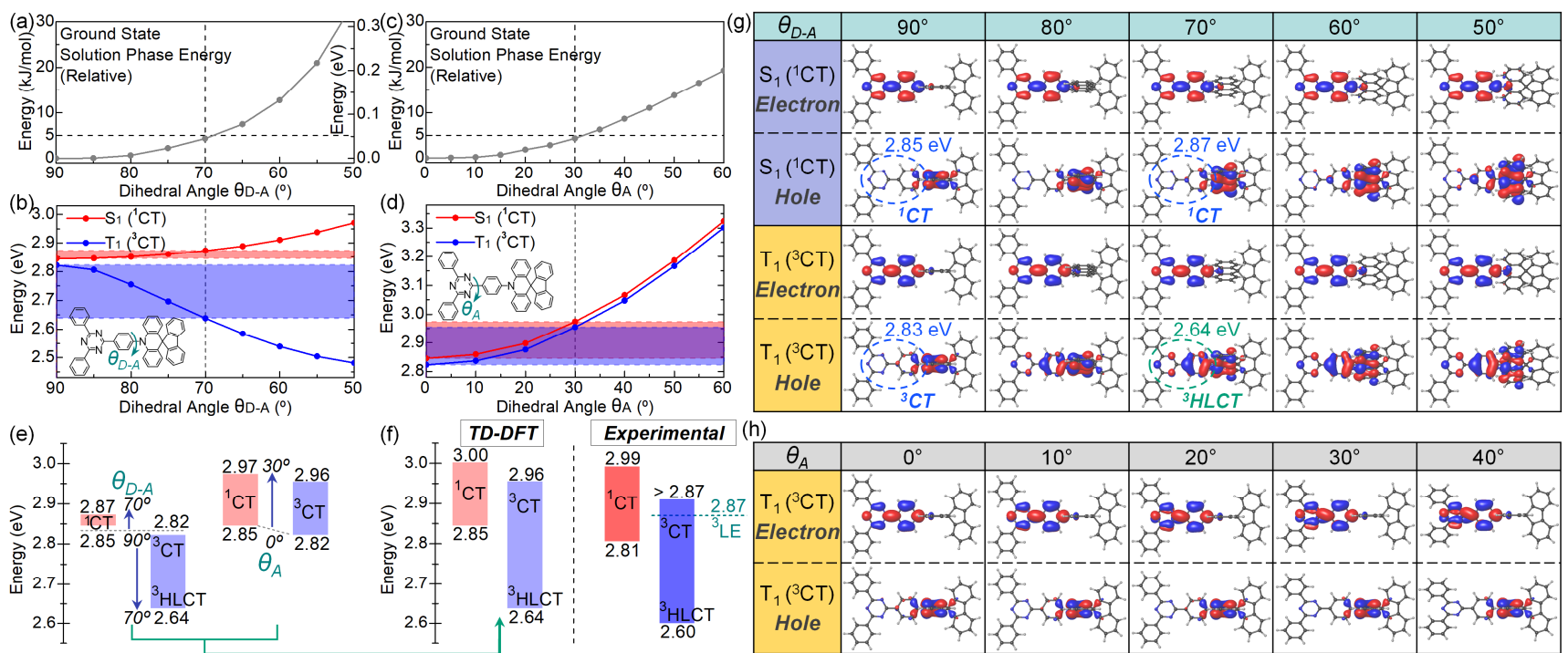

Figure 4 TD-DFT calculation of TPSA. (a)-(b) Ground state solution phase energy and $\mathrm{S}_{1}\left({ }^{1} \mathrm{CT}\right) / \mathrm{T}_{1}\left({ }^{3} \mathrm{CT}\right)$ energy of TPSA as a function of $\theta_{\mathrm{D}-\mathrm{A} \text {. }}$ (c)-(d) Ground state solution phase energy and $\mathrm{S}_{1}\left({ }^{1} \mathrm{CT}\right) / \mathrm{T}_{1}\left({ }^{3} \mathrm{CT}\right)$ energy of TPSA as a function of $\theta_{\mathrm{A}}$. (e) Energy distribution of TPSA obtained from (b) and (d). (f) Comparison of energy distribution of TPSA obtained from TD-DFT calculation and experiment. (g) NTOs of $\mathrm{S}_{1}\left({ }^{1} \mathrm{CT}\right)$ and $\mathrm{T}_{1}\left({ }^{3} \mathrm{CT}\right)$ states of TPSA as a function of $\theta_{\mathrm{D}-\mathrm{A} \text {. }}$ (h) NTOs of $\mathrm{S}_{1}\left({ }^{1} \mathrm{CT}\right)$ and $\mathrm{T}_{1}\left({ }^{3} \mathrm{CT}\right)$ states of TPSA as a function of $\theta_{\mathrm{A}}$.

addition, the calculation underestimated the energies of $S_{1}$ and $\mathrm{T}_{1}$ compared to the experimental values. Another report $^{50}$ showed a reasonable tendency of the $S_{1}$ and $T_{1}$ energies as a function of the dihedral angle: increase of $S_{1}$ and decrease of $\mathrm{T}_{1}$ by the decrease of the dihedral angle. However, the results also showed underestimated $\mathrm{S}_{1}$ and $\mathrm{T}_{1}$ energies due to the B3LYP functional. There is another report $^{51}$ of $S_{1}$ and $T_{1}$ energies of a TADF molecule as a function of the dihedral angle claiming that $\Delta \mathrm{Ess}_{\mathrm{s}}$ can be negative. However, the report used TD-DFT for the calculation of $\mathrm{S}_{1}$ energy while using unrestricted DFT (UDFT) for the calculation of $\mathrm{T}_{1}$ energy, which was later pointed out that the calculation result is an artifact of the mixed level of theories. ${ }^{52}$ Meanwhile, there are several reports that the long-range corrected (LRC) functionals with modified range separation parameter $(\omega)$ predicts singlet charge-transfer energies of TADF molecules better than global hybrid functionals. ${ }^{44,48,61}$ Therefore, we set four criteria for the TDDFT calculation of ${ }^{1} \mathrm{CT}$ and ${ }^{3} \mathrm{CT}$ states as a function of the dihedral angle. (1) Long range corrected functional should be employed. (2) The functional should have appropriate amount of exact exchange for reasonable tendency of energies and transition orbitals of ${ }^{3} \mathrm{CT}$ state. (3) The same level of theory for ${ }^{1} \mathrm{CT}$ and ${ }^{3} \mathrm{CT}$ should be used. (4) The energies from the calculation should be comparable to the energies determined from experiments.

To find optimal functional for the TD-DFT calculation, various functionals were tested with TPSA (Figure. 3a, S7S8). For the geometries of the dihedral angle scan, the following optimization was used: The ${ }^{1} \mathrm{CT}$ state geometry of TPSA was optimized by TD-DFT optimization of Gaussian0962 software with CAM-B3LYP functional. The DA dihedral angle of the optimized ${ }^{1} \mathrm{CT}$ geometry is $\sim 90^{\circ}$. Then, the optimized ${ }^{1} \mathrm{CT}$ geometry is used as an input geometry for the TD-DFT optimization (M06-2X) of the ${ }^{3} \mathrm{CT}$ state at a fixed D-A angle. During all optimizations, the state in interest is constantly monitored to check whether the TDDFT calculation is appropriate. Finally, the D-A angle of the output of the second geometry optimization is manually modified from $90^{\circ}$ to $50^{\circ}$ for the single point calculation of the dihedral angle scan of ${ }^{1} \mathrm{CT}$ and ${ }^{3} \mathrm{CT}$ energies. The single point calculations were performed with Jaguar (Materials Science Suite, Schrödinger). ${ }^{63}$ The amounts of exact exchange for the various functionals tested for the dihedral scan are shown in Figure 3a and S7. The tendency of the change of ${ }^{1} \mathrm{CT}$ and ${ }^{3} \mathrm{CT}$ energies vary by the amount of exact exchange. The energies of ${ }^{1} \mathrm{CT}$ and ${ }^{3} \mathrm{CT}$ of charge-transfer type molecules are determined by competition of stabilization of the charge-transfer state and energy minimization by the exchange interaction of the electrons participating in the charge-transfer state. In singlet CT $\left({ }^{1} \mathrm{CT}\right)$ state, the stabilization of charge-transfer state is dominant due to the opposite spin states of the electrons involved in the transition. Consequently, the donor and acceptor prefer orthogonal geometry to minimize the potential energy by separating the transition orbitals. On the other hand, the spin states of the electrons involved in the transition of the ${ }^{3} \mathrm{CT}$ state are the same. The energy minimization by exchange interaction is dominant in ${ }^{3} \mathrm{CT}$ states and it leads to a less tilted geometry with increased overlap between transition orbitals. Hence, the ${ }^{1} \mathrm{CT}$ energy should decrease and ${ }^{3} \mathrm{CT}$ energy should increase by the increase of D-A dihedral angle. The calculation result of the B3LYP functional shows almost no change of ${ }^{3} \mathrm{CT}$ energy as a function of the dihedral angle due to the small amount of exact exchange (20\%) (Figure 3a). The M06 functional with $27 \%$ global exact exchange shows better tendency of ${ }^{3} \mathrm{CT}$ energy, but the CT energies are still too small compared to experimental energies (Figure 2d). On the other hand, calculation results by functionals with larger amount of exact exchange such as CAM-B3LYP, $\omega$ B97XD*, and M06-HF show a significant problem in predicting ${ }^{3} \mathrm{CT}$ energies: the ${ }^{3} \mathrm{CT}$ states are separated into two states indicated in Figure $3 \mathrm{a}$ and S7 as " $\mathrm{T}_{1}$ " and "lowest ${ }^{3} \mathrm{CT} /{ }^{3} \mathrm{HLCT}$ above $\mathrm{T}_{1}$ ". Energies of " $\mathrm{T}_{1}$ " are significantly lower than ${ }^{1} \mathrm{CT}$ energies and energies of "lowest ${ }^{3} \mathrm{CT} /{ }^{3} \mathrm{HLCT}$ above $\mathrm{T}_{1}$ " are higher than energies of ${ }^{1} \mathrm{CT}$ states and somewhere in the middle of those energies seems to be reasonable for correct ${ }^{3} \mathrm{CT}$ 
energies. In addition, the separation of ${ }^{3} \mathrm{CT}$ states becomes more significant at lower dihedral angles. The NTOs of TPSA at $\theta_{\mathrm{D}-\mathrm{A}}=70^{\circ}$ calculated with CAM-B3LYP functional are shown in Figure $3 \mathrm{~b}$ to describe the separation of ${ }^{3} \mathrm{CT}$ states. At $\theta_{D-A}=90^{\circ}$ and $80^{\circ}, \mathrm{T}_{1}$ states are ${ }^{3} \mathrm{CT}$ states and the NTOs are similar to those of $S_{1}\left({ }^{1} \mathrm{CT}\right)$ states (Figure $S 9$ ). When $\theta_{\mathrm{D}-\mathrm{A}}$ is $70^{\circ}$ or smaller, $\mathrm{T}_{1}$ states are calculated as ${ }^{3} \mathrm{LE}$ states and the lowest ${ }^{3} \mathrm{CT} /{ }^{3} \mathrm{HLCT}$ states become higher than the ${ }^{1} \mathrm{CT}$ states (Figure $3 \mathrm{~b}$ ). This phenomenon is so-called "triplet instability" stemming from the exchange-interactionsensitive nature of triplet states. ${ }^{53-56,60}$ To solve this problem, UDFT calculation or Tamm-Dancoff approximation that excludes some terms in the equation of TD-DFT that causes triplet instability are often employed for the calculation of triplet states. ${ }^{48,53,55}$ However, instead of employing UDFT or Tamm-Dancoff approximation, we tried optimizing LRC functionals for TD-DFT calculation to solve the triplet instability. Figure $3 \mathrm{c}$ and $3 \mathrm{~d}$ show the amount of exact exchange and calculation results of the LRC functionals we tested. The amount of exact exchange of LRC functionals is determined by the following equation: $\boldsymbol{X}\left(r_{12}\right)$ $=\alpha+\beta \operatorname{erf}\left(\omega r_{12}\right)$, where $r_{12}$ is the interelectron distance, $\alpha$ is the short-range $\left(r_{12}=0\right)$ exact exchange, $\beta$ is the long-range $\left(r_{12}=\infty\right)$ exact exchange, and $\omega$ is the range separation parameter (Figure 3c). ${ }^{64}$ The $\omega$ of each functionals were modified to match the $S_{1}$ energy at $\theta_{D-A}=0^{\circ}$ to the $S_{1}$ of TPSA determined from the experiment. The modified $\omega$ for $\omega$ PBE$^{*}, \omega \mathrm{PBEH}^{*}, \omega \mathrm{B}^{*} \mathrm{X}^{*}, \omega \mathrm{B} 97 \mathrm{XD}^{*}$, and $\mathrm{M} 11^{*}$ functionals ${ }^{65-68}$ were $0.195,0.142,0.150,0.13$, and 0.10 , respectively. The calculation results are shown in Figure $3 \mathrm{~d}$. All functionals showed almost the same energy level and tendency for $S_{1}\left({ }^{1} \mathrm{CT}\right)$ states at all D-A dihedral angles. However, all functionals except M11* exhibited triplet instability for ${ }^{3} \mathrm{CT}$ calculations (Figure $3 \mathrm{~d}$ and S10-14). For the calculation results of $\omega \mathrm{PBE}^{*}, \omega \mathrm{PBEH}^{*}, \omega \mathrm{B} 97 \mathrm{X}^{*}$, and $\omega \mathrm{B} 97 \mathrm{XD}^{*}$, the charge-transfer type triplet states were separated into two states with an NTO configuration of larger hole-electron overlap and smaller hole-electron overlap. For example, the calculation result of $\omega \mathrm{PBEH}^{*}$ at $80^{\circ}$, the $\mathrm{T}_{1}$ state is calculated as ${ }^{3} \mathrm{LE}$ and the lowest ${ }^{3} \mathrm{CT}$ is even higher than the lowest ${ }^{1} \mathrm{CT}$ (Figure $3 \mathrm{~d}$ ). On the other hand, the calculations performed with M11* show robust ${ }^{3} \mathrm{CT} /{ }^{3} \mathrm{HLCT}$ results at all dihedral angles and the NTO configurations (Figure $3 \mathrm{~d}$ and S14) and ${ }^{3} \mathrm{CT}$ energies were consistent with the explanations mentioned above. The difference between the M11* and the other functionals are the exchange amount at the short range $\left(r_{12}<3 \mathrm{bohr}\right)$ and mid-range ( 3 bohr $<r_{12}<15$ bohr). Due to the small $\alpha$ and large $\omega$ of $\omega \operatorname{PBE}^{*}(\alpha=0), \quad \omega \operatorname{PBEH}^{*}(\alpha=0.2)$, $\omega B 97 X^{*}(\alpha=0.1577)$, and $\omega B 97 X D^{*}(\alpha=0.222)$, the amount of exact exchange rapidly increases in the distance range of the electron displacement involved in the charge-transfer transition. Consequently, the exchange interaction between the electrons involved in the ${ }^{3} \mathrm{CT}$ transition is not calculated properly, leading to the triplet instabilities. Therefore, the M11 $(\alpha=0.428)$ functional with modified $\omega$ of 0.10 were chosen for the TD-DFT single point calculations of the dihedral angle scan of ${ }^{1} \mathrm{CT} /{ }^{3} \mathrm{CT}$ states of TPSA.

Not only the D-A dihedral angle $\left(\theta_{\mathrm{D}-\mathrm{A}}\right)$ but also the dihedral angle of triazine-linker $\left(\theta_{\mathrm{A}}\right)$ of the TADF molecules can have distribution in a solid matrix (Note that the change of dihedral angles between the triazine and the two peripheral phenyls linked to the triazine has a negligible effect to the energies and configuration of the CT states of TPSA, Figure S15). Hence, the dihedral angle scan of $\theta_{\mathrm{A}}$ in TPSA was also calculated (Figure S16, S17). The energies of $\mathrm{S}_{1}\left({ }^{1} \mathrm{CT}\right)$ and $\mathrm{T}_{1}\left({ }^{3} \mathrm{CT} /{ }^{3} \mathrm{HLCT}\right)$ states and the ground state solution phase energies (potential energies, B3LYP, PCMtoluene) of TPSA are shown in Figure 4a-d. To investigate the energy distribution of TADF molecules generated by the distribution of $\theta_{A}$ and $\theta_{D-A}$, the actual proportion of each conformations should be considered. However, the conformational distribution of real molecules is affected by many complicated factors such as conformational energy barrier, the cooling rate of solution, solvent molecule, the crystal structure of the solvent molecule, and temperature. We leave the consideration of those factors for future work. In this study, we focus on the upper/lower limit of CT energies to reproduce the electronic band structure of CT states of TPSA. Therefore, we considered the energy distribution of $S_{1} / T_{1}$ energies stemming from the ground state solution phase energy distribution of $5 \mathrm{~kJ} / \mathrm{mol}$ (note that thermal energy at $300 \mathrm{~K}$ is $2.5 \mathrm{~kJ} / \mathrm{mol}$ ), which leads to the good prediction of ${ }^{1} \mathrm{CT}$ energy distribution of TPSA (Figure 4f). If the ground state solution phase energy of TPSA is limited for $5 \mathrm{~kJ} / \mathrm{mol}$, the dihedral angles lie within $70^{\circ} \leq \theta_{D-A} \leq 90^{\circ}$ and $0^{\circ} \leq \theta_{A} \leq 30^{\circ}$, respectively (Figure $\left.4 a, c\right)$. By applying the range limit of the dihedral angles to the TDDFT calculation of $S_{1} / T_{1}$ energies, the energy distribution of $\mathrm{S}_{1} / \mathrm{T}_{1}$ states can be obtained (Figure $4 \mathrm{~b}, \mathrm{~d}$ ). The energy distributions of excited states induced by $\theta_{D-A}$ and $\theta_{A}$ are shown in Figure 4e and combined to one for the comparison with experimentally determined energy distribution of TPSA (Figure 4f). Although the proportion of each conformer are not considered in the calculation for CT energy distributions, the calculation results and experimentally determined energy distribution are quite consistent. The upper/lower limits of the energy distribution of ${ }^{1} \mathrm{CT}$ and ${ }^{3} \mathrm{CT}$ match well with the experiment (Note that the highest ${ }^{3} \mathrm{CT}$ energy cannot be measured experimentally because all ${ }^{3} \mathrm{CT}$ states with energies higher than $2.87 \mathrm{eV}$ would be transferred to the ${ }^{3} \mathrm{LE}$ state via ITET). Moreover, the overlap of experimentally determined ${ }^{1} \mathrm{CT} /{ }^{3} \mathrm{CT}$ energy bands is reproduced with the theoretical calculation by combining the energy distribution induced by the distributions of $\theta_{\mathrm{D}-\mathrm{A}}$ and $\theta_{\mathrm{A}}$. If only the $\theta_{\mathrm{D}-\mathrm{A}}$ is considered, the singlet energy of TPSA for every conformer is always higher than the triplet energy of all conformers of TPSA. However, if $\theta_{\mathrm{A}}$ is also considered, the ${ }^{3} \mathrm{CT}$ energy of a certain conformer can be higher than the ${ }^{1} \mathrm{CT}$ energy of another conformer, leading to an overlap of the electronic band structure of CT states. NTOs of $\mathrm{S}_{1}\left({ }^{1} \mathrm{CT}\right) / \mathrm{T}_{1}\left({ }^{3} \mathrm{CT}\right)$ states of TPSA as a function of $\theta_{D-A}$ and $\theta_{A}$ are calculated to investigate the effect of dihedral angles to the CT state energies and excited state configurations (Figure 4g-h, S1617). At $\theta_{D-A}=90^{\circ}$, the NTOs for the $S_{1}\left({ }^{1} \mathrm{CT}\right)$ and $T_{1}\left({ }^{3} \mathrm{CT}\right)$ states of TPSA are almost the same. The NTOs for the $S_{1}\left({ }^{1} \mathrm{CT}\right)$ state at $\theta_{D-A}=70^{\circ}$ are not much different from those at $90^{\circ}$. However, the $\mathrm{T}_{1}$ state at $70^{\circ}$ becomes ${ }^{3}$ HLCT state with an increased overlap of the hole and electron transition orbitals at the phenyl linker, leading to a decreased $\mathrm{T}_{1}$ energy of $2.64 \mathrm{eV}$ from $2.83 \mathrm{eV}$ at $\theta_{\mathrm{D}-\mathrm{A}}=90^{\circ}$. On the other hand, as the $\theta_{\mathrm{A}}$ increase from $0^{\circ}$ to $30^{\circ}$, both the NTOs of $\mathrm{S}_{1}$ and $\mathrm{T}_{1}$ state shows a consistent configuration of charge- 
transfer type state due to the fixed linker-donor angle of $90^{\circ}$. However, the ${ }^{1} \mathrm{CT}$ and ${ }^{3} \mathrm{CT}$ energies increase with decreasing $\theta_{\mathrm{A}}$ due to the increased LUMO level by reduced conjugation between the linker and triazine. Changes in the transition orbital configurations and energies of the CT state resulting from the change of the dihedral angles would give a significant effect on the RISC process of the TADF molecules which will be further discussed in our future reports.

\section{CONCLUSION}

We determined the energy distribution of triplet and singlet charge-transfer states of the TADF emitter in the frozen matrix and reproduced the experimentally obtained electronic band with the theoretical calculation. From the total phosphorescence measurement of TPSA in frozen toluene at $77 \mathrm{~K}$, three types of triplet emission including ${ }^{3} \mathrm{CT}$, ${ }^{3} \mathrm{HLCT}$, and ${ }^{3} \mathrm{LE}$ was observed, leading to the determination of energy distribution of ${ }^{3} \mathrm{CT}$ state. Combined with the ${ }^{1} \mathrm{CT}$ energy distribution determined from time-resolved PL measurement of fluorescence and TADF, a unique electronic band structure with an overlap of singlet and triplet energies was obtained. To investigate the origin of the energy distribution of the CT state of TPSA, the dihedral angle scan of the ${ }^{3} \mathrm{CT}$ state was tested using functionals with various amounts of exact exchange and we found that the long-range corrected functional M11 with optimized rangeseparation parameter shows robust ${ }^{3} \mathrm{CT}$ calculation results without triplet instability. We limited the range of $\theta_{D-A}$ and $\theta_{\mathrm{A}}$ based on solution phase energy of TPSA and performed TD-DFT calculation for ${ }^{1} \mathrm{CT} /{ }^{3} \mathrm{CT}$ energies with optimized M11* functional. The energy distribution, band structure, and excited state configuration obtained from TD-DFT matched very well with the experimental data. Our results show that the conformational distribution of TADF molecules in a solid matrix must be considered for further development of efficient and stable TADF emitters.

\section{ASSOCIATED CONTENT}

\section{Supporting Information.}

The Supporting Information is available free of charge on the ACS Publications website.

\section{AUTHOR INFORMATION}

\section{Corresponding Author}

Jang-Joo Kim: ORCID 0000-0002-3500-7494

\section{Notes}

The authors declare no competing financial interest.

\section{ACKNOWLEDGMENT}

This work was supported by the Industrial Strategic Technology Development Program (10079671) funded by the Ministry of Trade, Industry, and Energy (MOTIE, Korea). We thank Prof. Yun-Hi Kim of GNU for providing TPSA.

\section{REFERENCES}

(1) Baldo, M. A.; O’Brien, D. F.; Thompson, M. E.; Forrest, S. R. Excitonic Singlet-Triplet Ratio in a Semiconducting Organic Thin Film. Phys. Rev. B 1999, 60, 14422-14428.

(2) Endo, A.; Sato, K.; Yoshimura, K.; Kai, T.; Kawada, A. Miyazaki, H.; Adachi, C. Efficient Up-Conversion of Triplet Excitons into a Singlet State and Its Application for Organic Light Emitting Diodes. Appl. Phys. Lett. 2011, 98, 083302.
(3) Yang, Z.; Mao, Z.; Xie, Z.; Zhang, Y.; Liu, S.; Zhao, J.; Xu, J.; Chi, Z.; Aldred, M. P. Recent Advances in Organic Thermally Activated Delayed Fluorescence Materials. Chem. Soc. Rev. 2017, 46, 915-1016.

(4) Wong, M. Y.; Zysman-Colman, E. Purely Organic Thermally Activated Delayed Fluorescence Materials for Organic LightEmitting Diodes. Adv. Mater. 2017, 29, 1605444.

(5) Jeon, S. K.; Lee, H. L.; Yook, K. S.; Lee, J. Y. Recent Progress of the Lifetime of Organic Light-Emitting Diodes Based on Thermally Activated Delayed Fluorescent Material. Adv. Mater. 2019, 31, 1803524.

(6) Uoyama, H.; Goushi, K.; Shizu, K.; Nomura, H.; Adachi, C. Highly Efficient Organic Light-Emitting Diodes from Delayed Fluorescence. Nature 2012, 492, 234-238.

(7) Rajamalli, P.; Senthilkumar, N.; Huang, P.-Y.; Ren-Wu, C.-C.; Lin, H.-W.; Cheng, C.-H. New Molecular Design Concurrently Providing Superior Pure Blue, Thermally Activated Delayed Fluorescence and Optical Out-Coupling Efficiencies. J. Am. Chem. Soc. 2017, 139, 10948-10951.

(8) Zhang, Q.; Li, J.; Shizu, K.; Huang, S.; Hirata, S.; Miyazaki, H.; Adachi, C. Design of Efficient Thermally Activated Delayed Fluorescence Materials for Pure Blue Organic Light Emitting Diodes. J. Am. Chem. Soc. 2012, 134, 14706-14709.

(9) Hatakeyama, T.; Shiren, K.; Nakajima, K.; Nomura, S.; Nakatsuka, S.; Kinoshita, K.; Ni, J.; Ono, Y.; Ikuta, T. Ultrapure Blue Thermally Activated Delayed Fluorescence Molecules: Efficient HOMO-LUMO Separation by the Multiple Resonance Effect. Adv. Mater. 2016, 28, 2777-2781.

(10) Ahn, D. H.; Kim, S. W.; Lee, H.; Ko, I. J.; Karthik, D.; Lee, J. Y.; Kwon, J. H. Highly Efficient Blue Thermally Activated Delayed Fluorescence Emitters Based on Symmetrical and Rigid Oxygen-Bridged Boron Acceptors. Nat. Photonics 2019, $13,540-546$

(11) Zhang, D.; Cai, M.; Zhang, Y.; Zhang, D.; Duan, L. Sterically Shielded Blue Thermally Activated Delayed Fluorescence Emitters with Improved Efficiency and Stability. Mater. Horizons 2016, 3, 145-151.

(12) Noda, H.; Nakanotani, H.; Adachi, C. Excited State Engineering for Efficient Reverse Intersystem Crossing. Sci. Adv. 2018, 4, eaao6910.

(13) Chan, C.-Y.; Tanaka, M.; Nakanotani, H.; Adachi, C. Efficient and Stable Sky-Blue Delayed Fluorescence Organic LightEmitting Diodes with CIEy below 0.4. Nat. Commun. 2018, 9 , 5036.

Ai, Q.; Chai, J.; Lou, W.; Liu, T.; Wang, D.; Deng, C.; Wang, C.; Li, G.; Liu, X.; Liu, Z.; Zhang, Q. Efficient and Stable Organic Light-Emitting Diodes Employing Indolo[2,3- b ]IndoleBased Thermally Activated Delayed Fluorescence Emitters. ACS Appl. Mater. Interfaces 2020, 12, 6127-6136.

Deng, C.; Zheng, S.; Wang, D.; Yang, J.; Yue, Y.; Li, M.; Zhou, Y.; Niu, S.; Tao, L.; Tsuboi, T.; Zhang, Q. Improving the Stability of Green Thermally Activated Delayed Fluorescence OLEDs by Reducing the Excited-State Dipole Moment. J. Phys. Chem. C 2019, 123, 29875-29883.

(16) Inoue, M.; SereviLius, T.; Nakanotani, H.; Yoshida, K. Matsushima, T.; JuršLnas, S.; Adachi, C. Effect of Reverse Intersystem Crossing Rate to Suppress Efficiency Roll-off in Organic Light-Emitting Diodes with Thermally Activated Delayed Fluorescence Emitters. Chem. Phys. Lett. 2016, 644, 62-67.

(17) Lee, J.; Aizawa, N.; Yasuda, T. Isobenzofuranone- and Chromone-Based Blue Delayed Fluorescence Emitters with Low Efficiency Roll-Off in Organic Light-Emitting Diodes. Chem. Mater. 2017, 29, 8012-8020.

(18) Woo, S.-J.; Kim, Y.; Kwon, S.-K.; Kim, Y.-H.; Kim, J.-J. Phenazasiline/Spiroacridine Donor Combined with MethylSubstituted Linkers for Efficient Deep Blue Thermally Activated Delayed Fluorescence Emitters. ACS Appl. Mater. Interfaces 2019, 11, 7199-7207.

(19) Cui, L.-S.; Deng, Y.-L.; Tsang, D. P.-K.; Jiang, Z.-Q.; Zhang, Q.; Liao, L.-S.; Adachi, C. Controlling Synergistic Oxidation Processes for Efficient and Stable Blue Thermally Activated Delayed Fluorescence Devices. Adv. Mater. 2016, 28, 76207625. 
(20) Nasu, K.; Nakagawa, T.; Nomura, H.; Lin, C.-I.; Cheng, C.-H. Tseng, M.-R.; Yasuda, T.; Adachi, C. A Highly Luminescent Spiro-Anthracenone-Based Organic Light-Emitting Diode Exhibiting Thermally Activated Delayed Fluorescence. Chem. Commun. 2013, 49, 10385

(21) Nakagawa, T.; Ku, S.-Y.; Wong, K.-T.; Adachi, C. Electroluminescence Based on Thermally Activated Delayed Fluorescence Generated by a Spirobifluorene DonorAcceptor Structure. Chem. Commun. 2012, 48, 9580.

(22) Lin, T.-A.; Chatterjee, T.; Tsai, W.-L.; Lee, W.-K.; Wu, M.-J.; Jiao, M.; Pan, K.-C.; Yi, C.-L.; Chung, C.-L.; Wong, K.-T.; Wu, C.C. Sky-Blue Organic Light Emitting Diode with 37\% Externa Quantum Efficiency Using Thermally Activated Delayed Fluorescence from Spiroacridine-Triazine Hybrid. $A d v$. Mater. 2016, 28, 6976-6983.

(23) Oh, C. S.; Pereira, D. D. S.; Han, S. H.; Park, H.-J.; Higginbotham, H. F.; Monkman, A. P.; Lee, J. Y. Dihedral Angle Control of Blue Thermally Activated Delayed Fluorescent Emitters through Donor Substitution Position for Efficient Reverse Intersystem Crossing. ACS Appl. Mater. Interfaces 2018, 10, 35420-35429.

(24) Cui, L.-S.; Nomura, H.; Geng, Y.; Kim, J. U.; Nakanotani, H.; Adachi, C. Controlling Singlet-Triplet Energy Splitting for Deep-Blue Thermally Activated Delayed Fluorescence Emitters. Angew. Chemie 2017, 129, 1593-1597.

(25) Gibson, J.; Monkman, A. P.; Penfold, T. J. The Importance of Vibronic Coupling for Efficient Reverse Intersystem Crossing in Thermally Activated Delayed Fluorescence Molecules. ChemPhysChem 2016, No. 1, 2956-2961.

(26) Kaji, H.; Suzuki, H.; Fukushima, T.; Shizu, K.; Suzuki, K.; Kubo, S.; Komino, T.; Oiwa, H.; Suzuki, F.; Wakamiya, A.; Murata, Y.; Adachi, C. Purely Organic Electroluminescent Material Realizing 100\% Conversion from Electricity to Light. Nat. Commun. 2015, 6, 8476.

(27) Higginbotham, H. F.; Yi, C. L.; Monkman, A. P.; Wong, K. T. Effects of Ortho-Phenyl Substitution on the RISC Rate of D-A Type TADF Molecules. J. Phys. Chem. C 2018, 122, 76277634.

(28) Serevičius, T.; Skaisgiris, R.; Dodonova, J.; Jagintavičius, L.; Banevičius, D.; Kazlauskas, K.; Tumkevičius, S.; Juršènas, S. Achieving Submicrosecond Thermally Activated Delayed Fluorescence Lifetime and Highly Efficient

Electroluminescence by Fine-Tuning of the PhenoxazinePyrimidine Structure. ACS Appl. Mater. Interfaces 2020, 12 10727-10736.

(29) Vázquez, R. J.; Yun, J. H.; Muthike, A. K.; Howell, M.; Kim, H.; Madu, I. K.; Kim, T.; Zimmerman, P.; Lee, J. Y.; III, T. G. New Direct Approach for Determining the Reverse Intersystem Crossing Rate in Organic Thermally Activated Delayed Fluorescent (TADF) Emitters. J. Am. Chem. Soc. 2020, 142 8074-8079.

(30) Noda, H.; Chen, X.-K.; Nakanotani, H.; Hosokai, T.; Miyajima, M.; Notsuka, N.; Kashima, Y.; Brédas, J.-L.; Adachi, C. Critical Role of Intermediate Electronic States for Spin-Flip Processes in Charge-Transfer-Type Organic Molecules with Multiple Donors and Acceptors. Nat. Mater. 2019, 18, 10841090.

(31) Cui, L.-S.; Gillett, A. J.; Zhang, S.-F.; Ye, H.; Liu, Y.; Chen, X.-K.; Lin, Z.-S.; Evans, E. W.; Myers, W. K.; Ronson, T. K.; Nakanotani, H.; Reineke, S.; Bredas, J.-L.; Adachi, C.; Friend, R. H. Fast Spin-Flip Enables Efficient and Stable Organic Electroluminescence from Charge-Transfer States. Nat. Photonics 2020, 14, 636-642.

(32) Hosokai, T.; Matsuzaki, H.; Nakanotani, H.; Tokumaru, K.; Tsutsui, T.; Furube, A.; Nasu, K.; Nomura, H.; Yahiro, M.; Adachi, C. Evidence and Mechanism of Efficient Thermally Activated Delayed Fluorescence Promoted by Delocalized Excited States. Sci. Adv. 2017, 3, e1603282.

(33) Dias, F. B.; Santos, J.; Graves, D. R.; Data, P.; Nobuyasu, R. S.; Fox, M. A.; Batsanov, A. S.; Palmeira, T.; Berberan-Santos, M N.; Bryce, M. R.; Monkman, A. P. The Role of Local Triplet Excited States and D-A Relative Orientation in Thermally Activated Delayed Fluorescence: Photophysics and Devices. Adv. Sci. 2016, 3, 1600080.
(34) Etherington, M. K.; Gibson, J.; Higginbotham, H. F.; Penfold, T. J.; Monkman, A. P. Revealing the Spin-Vibronic Coupling Mechanism of Thermally Activated Delayed Fluorescence. Nat. Commun. 2016, 7, 13680.

(35) Weissenseel, S.; Drigo, N. A.; Kudriashova, L. G.; Schmid, M.; Morgenstern, T.; Lin, K.-H.; Prlj, A.; Corminboeuf, C.; Sperlich A.; Brütting, W.; Nazeeruddin, M. K.; Dyakonov, V. Getting the Right Twist: Influence of Donor-Acceptor Dihedral Angle on Exciton Kinetics and Singlet-Triplet Gap in Deep Blue Thermally Activated Delayed Fluorescence Emitter. J. Phys. Chem. C 2019, 123, 27778-27784.

(36) Woo, S.-J.; Ha, Y. H.; Kim, Y.-H.; Kim, J.-J. Effect of OrthoBiphenyl Substitution on the Excited State Dynamics of Multi-Carbazole TADF Molecule. J. Mater. Chem. C 2020, 8

(37) Kreiza, G.; Banevičius, D.; Jovaišaitè, J.; Maleckaitè, K. Gudeika, D.; Volyniuk, D.; Gražulevičius, J. V.; Juršènas, S.; Kazlauskas, K. Suppression of Benzophenone-Induced Triplet Quenching for Enhanced TADF Performance. J. Mater. Chem. C 2019, 7, 11522-11531.

(38) Zhang, Q.; Kuwabara, H.; Potscavage, W. J.; Huang, S.; Hatae, Y.; Shibata, T.; Adachi, C. Anthraquinone-Based Intramolecular Charge-Transfer Compounds: Computational Molecular Design, Thermally Activated Delayed Fluorescence, and Highly Efficient Red Electroluminescence. J. Am. Chem. Soc. 2014, 136, 18070-18081.

(39) Méhes, G.; Goushi, K.; Potscavage, W. J.; Adachi, C. Influence of Host Matrix on Thermally-Activated Delayed Fluorescence: Effects on Emission Lifetime, Photoluminescence Quantum Yield, and Device Performance. Org. Electron. 2014, 15, 2027-2037.

(40) Serevičius, T.; Skaisgiris, R.; Dodonova, J.; Jagintavičius, L.; Bucevičius, J.; Kazlauskas, K.; Juršènas, S.; Tumkevičius, S. Emission Wavelength Dependence on the RISC Rate in TADF Compounds with Large Conformational Disorder. Chem. Commun. 2019, 55, 1975-1978.

(41) Serevičius, T.; Skaisgiris, R.; Dodonova, J.; Kazlauskas, K.; Juršènas, S.; Tumkevičius, S. Minimization of Solid-State Conformational Disorder in Donor-Acceptor TADF Compounds. Phys. Chem. Chem. Phys. 2020, 22, 265-272. Serevičius, T.; Skaisgiris, R.; Fiodorova, I.; Steckis, V.; Dodonova, J.; Banevičius, D.; Kazlauskas, K.; Juršènas, S.; Tumkevičius, S. Achieving Efficient Deep-Blue TADF in Carbazole-Pyrimidine Compounds. Org. Electron. 2020, 82, 105723.

(43) Montanaro, S.; Gillett, A. J.; Feldmann, S.; Evans, E. W.; Plasser, F.; Friend, R. H.; Wright, I. A. Red-Shifted Delayed Fluorescence at the Expense of Photoluminescence Quantum Efficiency - an Intramolecular Charge-Transfer Molecule Based on a Benzodithiophene-4,8-Dione Acceptor. Phys. Chem. Chem. Phys. 2019, 21, 10580-10586.

(44) Shee, J.; Head-Gordon, M. Predicting Excitation Energies of Twisted Intramolecular Charge-Transfer States with the Time-Dependent Density Functional Theory: Comparison with Experimental Measurements in the Gas Phase and Solvents Ranging from Hexanes to Acetonitrile. J. Chem. Theory Comput. 2020, acs.jctc.0c00635.

(45) Cho, E.; Coropceanu, V.; Brédas, J.-L. Electronic Structure of Multicomponent Organic Molecular Materials: Evaluation of Range-Separated Hybrid Functionals. J. Chem. Theory Comput. 2020, 16, 3712-3719.

(46) Samanta, P. K.; Kim, D.; Coropceanu, V.; Brédas, J.-L. UpConversion Intersystem Crossing Rates in Organic Emitters for Thermally Activated Delayed Fluorescence: Impact of the Nature of Singlet vs Triplet Excited States. J. Am. Chem. Soc. 2017, 139, 4042-4051.

(47) Scholz, R.; Kleine, P.; Lygaitis, R.; Popp, L.; Lenk, S.; Etherington, M. K.; Monkman, A. P.; Reineke, S. Investigation of Thermally Activated Delayed Fluorescence from a DonorAcceptor Compound with Time-Resolved Fluorescence and Density Functional Theory Applying an Optimally Tuned Range-Separated Hybrid Functional. J. Phys. Chem. A 2020, 124, 1535-1553.

(48) Sun, H.; Zhong, C.; Brédas, J.-L. Reliable Prediction with Tuned Range-Separated Functionals of the Singlet-Triplet 
Gap in Organic Emitters for Thermally Activated Delayed Fluorescence. J. Chem. Theory Comput. 2015, 11, 3851-3858.

(49) Huang, S.; Zhang, Q.; Shiota, Y.; Nakagawa, T.; Kuwabara, K.; Yoshizawa, K.; Adachi, C. Computational Prediction for Singlet- and Triplet-Transition Energies of Charge-Transfer Compounds. J. Chem. Theory Comput. 2013, 9, 3872-3877.

(50) Hu, T.; Han, G.; Tu, Z.; Duan, R.; Yi, Y. Origin of High Efficiencies for Thermally Activated Delayed Fluorescence Organic Light-Emitting Diodes: Atomistic Insight into Molecular Orientation and Torsional Disorder. J. Phys. Chem C 2018, 122, 27191-27197.

(51) Di, D.; Romanov, A. S.; Yang, L.; Richter, J. M.; Rivett, J. P. H.; Jones, S.; Thomas, T. H.; Abdi Jalebi, M.; Friend, R. H.; Linnolahti, M.; Bochmann, M.; Credgington, D. HighPerformance Light-Emitting Diodes Based on CarbeneMetal-Amides. Science (80-. ). 2017, 356, 159-163.

(52) Penfold, T. J.; Gindensperger, E.; Daniel, C.; Marian, C. M. Spin-Vibronic Mechanism for Intersystem Crossing. Chem. Rev. 2018, 118, 6975-7025.

(53) Peach, M. J. G.; Williamson, M. J.; Tozer, D. J. Influence of Triplet Instabilities in TDDFT. J. Chem. Theory Comput. 2011 7, 3578-3585.

(54) Bousquet, D.; Fukuda, R.; Jacquemin, D.; Ciofini, I.; Adamo, C.; Ehara, M. Benchmark Study on the Triplet Excited-State Geometries and Phosphorescence Energies of Heterocyclic Compounds: Comparison Between TD-PBE0 and SAC-CI.J. Chem. Theory Comput. 2014, 10, 3969-3979.

(55) Rangel, T.; Hamed, S. M.; Bruneval, F.; Neaton, J. B. An Assessment of Low-Lying Excitation Energies and Triplet Instabilities of Organic Molecules with an Ab Initio BetheSalpeter Equation Approach and the Tamm-Dancoff Approximation. J. Chem. Phys. 2017, 146, 194108.

(56) Peach, M. J. G.; Tozer, D. J. Overcoming Low Orbital Overlap and Triplet Instability Problems in TDDFT. J. Phys. Chem. A 2012, 116, 9783-9789.

(57) Tran, J.; Olmsted, J. Intramolecular Triplet-Triplet Energy Transfer from Xanthene Dyes to an Anthryl Substituent. $J$ Photochem. Photobiol. A Chem. 1993, 71, 45-49.
(58) Wang, K.; Zheng, C.-J.; Liu, W.; Liang, K.; Shi, Y.-Z.; Tao, S.-L.; Lee, C.-S.; Ou, X.-M.; Zhang, X.-H. Avoiding Energy Loss on TADF Emitters: Controlling the Dual Conformations of D-A Structure Molecules Based on the Pseudoplanar Segments. Adv. Mater. 2017, 29, 1701476.

(59) Vydrov, O. A.; Heyd, J.; Krukau, A. V.; Scuseria, G. E Importance of Short-Range versus Long-Range Hartree-Fock Exchange for the Performance of Hybrid Density Functionals. J. Chem. Phys. 2006, 125, 074106.

(60) Jacquemin, D.; Perpète, E. A.; Ciofini, I.; Adamo, C. Assessment of Functionals for TD-DFT Calculations of Singlet-Triplet Transitions. J. Chem. Theory Comput. 2010, 6, 1532-1537.

(61) Tian, X.; Sun, H.; Zhang, Q.; Adachi, C. Theoretical Predication for Transition Energies of Thermally Activated Delayed Fluorescence Molecules. Chinese Chem. Lett. 2016, 27, 14451452.

(62) Gaussian 09, Gaussian, Inc., Wallingford CT 2009.

(63) Jaguar, Schrödinger, Inc., New York, NY 2020

(64) Yanai, T.; Tew, D. P.; Handy, N. C. A New Hybrid ExchangeCorrelation Functional Using the Coulomb-Attenuating Method (CAM-B3LYP). Chem. Phys. Lett. 2004, 393, 51-57.

(65) Vydrov, O. A.; Scuseria, G. E. Assessment of a Long-Range Corrected Hybrid Functional. J. Chem. Phys. 2006, 125.

(66) Chai, J.-D.; Head-Gordon, M. Long-Range Corrected Hybrid Density Functionals with Damped Atom-Atom Dispersion Corrections. Phys. Chem. Chem. Phys. 2008, 10, 6615.

(67) Peverati, R.; Truhlar, D. G. Performance of the M11 and M11 L Density Functionals for Calculations of Electronic Excitation Energies by Adiabatic Time-Dependent Density Functional Theory. Phys. Chem. Chem. Phys. 2012, 14, 11363

(68) Peverati, R.; Truhlar, D. G. Improving the Accuracy of Hybrid Meta-GGA Density Functionals by Range Separation. J. Phys. Chem. Lett. 2011, 2, 2810-2817. 


\section{TOC Figure}

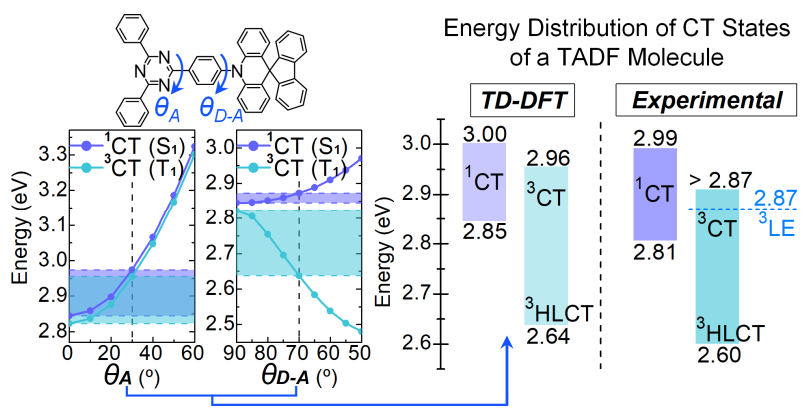

